Research Article

\title{
Current-Loop Control for the Pitching Axis of Aerial Cameras via an Improved ADRC
}

\author{
BingYou Liu, ${ }^{1,2}$ ChangAn Zhu, ${ }^{1}$ and XingZhong Guo ${ }^{2}$ \\ ${ }^{1}$ Department of Precision Machinery and Precision Instrumentation, University of Science and Technology of China, \\ Hefei 230027, China \\ ${ }^{2}$ Key Lab of Electric and Control of Anhui Province, Anhui Polytechnic University, Wuhu 241000, China
}

Correspondence should be addressed to BingYou Liu; lby009@mail.ustc.edu.cn

Received 13 July 2016; Revised 9 October 2016; Accepted 4 December 2016; Published 6 February 2017

Academic Editor: Rafael M. Herrera

Copyright (C) 2017 BingYou Liu et al. This is an open access article distributed under the Creative Commons Attribution License, which permits unrestricted use, distribution, and reproduction in any medium, provided the original work is properly cited.

\begin{abstract}
An improved active disturbance rejection controller (ADRC) is designed to eliminate the influences of the current-loop for the pitching axis control system of an aerial camera. The improved ADRC is composed of a tracking differentiator (TD), an improved extended state observer (ESO), an improved nonlinear state error feedback (NLSEF), and a disturbance compensation device (DCD). The TD is used to arrange transient process. The improved ESO is utilized to observe the state extended by nonlinear dynamics, model uncertainty, and external disturbances. Overtime variation of the current-loop can be predicted by the improved ESO. The improved NLSEF is adopted to restrain the residual errors of the current-loop. The DCD is used to compensate the overtime variation of the current-loop in real time. The improved ADRC is designed based on a new nonlinear function newfal $(\cdot)$. This function exhibits enhanced continuity and smoothness compared to previously available nonlinear functions. Thus, the new nonlinear function can effectively decrease the high-frequency flutter phenomenon. The improved ADRC exhibits improved control performance, and disturbances of the current-loop can be eliminated by the improved ADRC. Finally, simulation experiments are performed. Results show that the improved ADRC displayed better performance than the proportional integral (PI) control strategy and traditional ADRC.
\end{abstract}

\section{Introduction}

The pitching axis control system of an aerial camera adopts a servo control system with three loops, namely, currentloop, speed-loop, and position-loop. As an inner loop, the current-loop plays an important role in the pitching axis control system of an aerial camera [1, 2]. This loop should be controlled in real time to achieve good performance of the pitching axis control system. Therefore, the current-loop needs an improved control strategy. The current-loop control strategy has been extensively investigated globally, and several developments have been achieved. For example, Radwan and Mohamed [3] examined an improved vector control strategy, but the vector control strategy needs accurately known system parameters. Liu et al. [4] introduced current control strategy with an optimal variable structure that exhibits improved robustness. However, this control strategy cannot solve the jitter problem. Novak and Riener [5] examined an artificial intelligence control strategy, which does not rely on the mathematical model of the controlled object. However, this control strategy has a complex algorithm. Liu et al. [6] examined an adaptive current control technique with constant frequency based on clocked signal. This control strategy exhibits good static performance but weak restrain capacity for external disturbance. Several studies investigated a traditional active disturbance rejection control strategy, which exhibits certain anti-interference ability. In [7], Han introduced the evolution from PID to ADRC and the advantages of ADRC. In [8-10], Guo used active disturbance rejection control (ADRC) and sliding mode control (SMC) in the stabilization of the Euler-Bernoulli beam equation, onedimensional and multidimensional antistable wave equations with boundary input disturbance. In [11], Sira-Ramírez et al. presented an ADRC scheme for the angular velocity trajectory tracking task on a substantially perturbed, uncertain, and permanent magnet synchronous motor. An ADRC was designed to solve the trajectory tracking problem of a Delta robot with uncertain dynamical model [12]. The stabilization 
problem of a class of nonlinear systems with actuator saturation is investigated via ADRC [13]. An adaptive extended state observer- (AESO-) based ADRC is proposed to deal with the uncertainties and applied to the air-fuel ratio (AFR) control of gasoline engine, which has large nonlinear uncertainties due to the unknown speed change, fuel film dynamics, and so on [14]. The absolute stability of nonlinear ADRC for singleinput-single-output systems is analyzed by the circle criterion method [15]. In [16], an ADRC was applied to stabilization for lower triangular nonlinear systems with large uncertainties. Guo et al. [17] generalized the ADRC to uncertain nonlinear systems subject to external bounded stochastic disturbance described by an uncertain stochastic differential equation driven by white noise. Liu et al. [18] proposed a two-layer ADRC method with the compensation of estimated equivalent input disturbances (EID) for load frequency control (LFC) of multiarea interconnected power system. However, the high-frequency flutter phenomenon is not eliminated, and the anti-interference ability needs to be enhanced. The current-loop of the pitching axis control system of an aerial camera is controlled under weightlessness. Therefore, a small interference will result in a serious error. The control system requires an improved active disturbance rejection controller (ADRC) with strong anti-interference ability.

The improved ADRC is composed of a tracking differentiator (TD), an improved extended state observer (ESO), an improved nonlinear state error feedback (NLSEF), and a disturbance compensation device (DCD). In this study, the previously available nonlinear function of a traditional ADRC is improved to a new nonlinear function with better continuity and smoothness. Thus, the improved ADRC based on the new nonlinear function exhibits better antiinterference performance. On the one hand, the total disturbances are sufficiently estimated by the improved ESO, and the state of the system does not require direct observation. Therefore, the controllability of the system can be enhanced greatly. On the other hand, control efficiency is enhanced using the improved NLSEF to conduct nonlinear calculation of the proportion signal, differential signal, and integral signal of the error $[19,20]$. Extensive studies on the improved ADRC have resulted in important progress. Qi et al. [21] constructed a continuous and smooth ESO and disturbance rejection cascade decoupling control technology. An improved ADRC has been applied in a hypersonic vehicle and in the straight course error modeling of an electromagnetically controlled gyrocompass [22]. In [23], an improved ADRC based on the nonlinear arctangent function is used to reduce the observation noise in the traditional extended state observation of a system with output measurement noise. An improved ADRC is proposed to improve the tracking performance of the electromechanical actuator [24]. In [25], an improved ADRC is used to control an autonomous underwater vehicle. In [26], an enhanced ADRC is presented for a twin-rotor multi-input multioutput system with two degrees of freedom. However, few reports have focused on the use of improved ADRC in the current-loop control system of the pitching axis of an aerial camera.

The present study proposes an improved ADRC based on a new nonlinear function newfal(.). Then, the controller is applied to the current-loop control system of an aerial camera pitching axis. Simulations are conducted for cases adopting the PI control strategy, traditional ADRC, and improved ADRC. Finally, the simulation results are discussed.

\section{Mathematical Model}

The pitching axis of an aerial camera adopts a permanent magnet synchronous motor (PMSM) as an actuator. A control strategy of $i_{d}=0$ is used in the current-loop control system, where $i_{d}$ is the current of the direct axis. Therefore, the controlled object of the current-loop is $i_{q}$, where $i_{q}$ is the current of the quadrature axis. The current equation of the quadrature axis in a $(d, q)$ two-phase rotating coordinate system is given as follows:

$$
i_{q}=-\frac{R}{L} i_{q}-\frac{\psi_{f}}{L} \omega+\frac{1}{L} u_{q},
$$

where $R$ is the stator phase resistance, $L$ is the quadrature axis inductance, $\psi_{f}$ is the magnetic chain produced by the rotor permanent magnet, $u_{q}$ is the equivalent voltage of the quadrature axis, and $\omega$ is the angular speed of the motor rotor. The mathematical model of PMSM in a $(d, q)$ two-phase rotating coordinate system shows that the electromagnetic torque is directly controlled by $i_{q}$. Therefore, the precise control of the electromagnetic torque can be realized by controlling the current-loop. The expression $-\left(\psi_{f} / L\right) \omega$ represents a coupling effect in the current equation of the quadrature axis. The current-loop is a nonlinear system under the influence of the coupling effect. Several definitions are given as follows:

$$
\begin{aligned}
w(t) & =-\frac{\psi_{f}}{L} \omega, \\
f\left(i_{q}, t\right) & =-\frac{R}{L} i_{q}, \\
b & =\frac{1}{L}, \\
u & =u_{q} .
\end{aligned}
$$

Then, the current equation of the quadrature axis can be expressed as follows:

$$
\dot{i_{q}}=f\left(i_{q}, t\right)+w(t)+b u
$$

where $f\left(i_{q}, t\right)$ and $w(t)$ are the internal and external disturbances of the current-loop, respectively. The total disturbance of the current-loop is expressed as $a(t)=f\left(i_{q}, t\right)+w(t)$. Then, the current equation of quadrature axis is as follows:

$$
i_{q}=a(t)+b u
$$

Equation (4) is a typical equation used to design the improved ADRC.

\section{Improved ADRC Design for the Current-Loop}

3.1. Design of the New Nonlinear Function. The nonlinear function is the core of the ADRC. The following conditions 
should be fully considered in designing the nonlinear function. First, the nonlinear function should exhibit good convergence around the origin. Second, the value of the nonlinear function is 0 at the origin. Third, the nonlinear function should be continuous around the origin. The traditional ADRC adopts a nonlinear function fal $(\cdot)$, which can be expressed as follows:

$$
\operatorname{fal}(e, \alpha, \delta)=\left\{\begin{array}{ll}
|e|^{\alpha} \operatorname{sign}(e), & |e|>\delta \\
\frac{e}{\delta^{1-\alpha}}, & |e| \leq \delta,
\end{array} \quad \delta>0 .\right.
$$

The characteristics of fal $(\cdot)$ are given as follows. The value of $\alpha$ influences the nonlinearity degree of fal( $\cdot)$. The value of $\alpha$ is usually selected between 0 and 1 . The nonlinear function exhibits optimal nonlinearity when $\alpha=0$. The degree of linearity of $\mathrm{fal}(\cdot)$ is optimal when $\alpha=1$. The value of $\delta$ is the linear interval width of fal $(\cdot)$ and related to the error range of the system. When the input is an error signal, the system can achieve rapid stability by adjusting the parameter values of $\mathrm{fal}(\cdot)$. fal( $\cdot)$ is a nonderivable function, although this function is continuous. If the value of $\delta$ is too small, fal $(\cdot)$ will also cause the high-frequency flutter phenomenon. The value of $\delta$ is difficult to adjust, because the control performance of fal( $\cdot)$ is sensitive to the value of $\delta$. Thus, a new nonlinear function newfal $(\cdot)$ is designed. The proposed newfal $(\cdot)$ exhibits better convergence and continuity around the origin than fal $(\cdot)$. The expression of newfal $(\cdot)$ is calculated by the following steps.

When $|e|>\delta$, newfal $(\cdot)$ is expressed as newfal $(e, \alpha, \delta)=$ $|e|^{\alpha} \operatorname{sign}(e)$.

When $|e| \leq \delta$, the expression of newfal( $\cdot)$ is designed as an interpolation function form by multinomial and trigonometric functions and can be expressed as follows:

$$
\operatorname{newfal}(e, \alpha, \delta)=k_{1} e+k_{2} e^{2}+k_{3} \tan e
$$

Part three of the above expression is selected as tan $e$, but not $e^{3}$, because tan $e$ exhibits better convergence than $e^{3}$ around the origin. Then, newfal $(e, \alpha, \delta)$ can be given as follows:

$$
\operatorname{newfal}(e, \alpha, \delta)= \begin{cases}|e|^{\alpha} \operatorname{sign}(e), & |e|>\delta \\ k_{1} e+k_{2} e^{2}+k_{3} \tan e, & |e| \leq \delta\end{cases}
$$

where $k_{1}, k_{2}$, and $k_{3}$ are the function coefficients and can be calculated by the following steps.

In the range of $|e| \leq \delta$, the following expression is given to meet the continuous and derivable conditions:

$$
\begin{gathered}
\operatorname{newfal}(e, \alpha, \delta)=\delta^{\alpha}, \quad e=\delta, \\
\operatorname{newfal}(e, \alpha, \delta)=-\delta^{\alpha}, \quad e=-\delta, \\
\operatorname{newfal}^{\prime}(e, \alpha, \delta)=\alpha \delta^{\alpha}, \quad e= \pm \delta .
\end{gathered}
$$

The following expression can be obtained by (7) and (8),

$$
\begin{aligned}
k_{1} \delta+k_{2} \delta^{2}+k_{3} \tan \delta & =\delta^{\alpha}, \\
-k_{1} \delta+k_{2} \delta^{2}-k_{3} \tan \delta & =-\delta^{\alpha}, \\
k_{1}+2 k_{2} \delta+k_{3} \sec ^{2} \delta & =\alpha \delta^{\alpha} .
\end{aligned}
$$

The values of $k_{1}, k_{2}$, and $k_{3}$ can be calculated as follows:

$$
\begin{aligned}
& k_{1}=\frac{\delta^{\alpha}+\delta^{\alpha} \cdot \tan ^{2} \delta-\alpha \cdot \tan \delta \cdot \delta^{\alpha}}{\delta-\tan \delta+\delta \cdot \tan ^{2} \delta}, \\
& k_{2}=0, \\
& k_{3}=\frac{\alpha \cdot \delta^{\alpha+1}-\delta^{\alpha}}{\delta-\tan \delta+\delta \cdot \tan ^{2} \delta} .
\end{aligned}
$$

Thus, the expression of newfal $(e, \alpha, \delta)$ can be obtained as follows:

$$
\operatorname{newfal}(e, \alpha, \delta)= \begin{cases}|e|^{\alpha} \operatorname{sign}(e), & |e|>\delta \\ \frac{\delta^{\alpha}+\delta^{\alpha} \cdot \tan ^{2} \delta-\alpha \cdot \tan \delta \cdot \delta^{\alpha}}{\delta-\tan \delta+\delta \cdot \tan ^{2} \delta} e+\frac{\alpha \cdot \delta^{\alpha+1}-\delta^{\alpha}}{\delta-\tan \delta+\delta \cdot \tan ^{2} \delta} \tan e, & |e| \leq \delta\end{cases}
$$

Simulations are performed using the two nonlinear functions under $\alpha=0.05, \delta=0.06$. The curves of the function are shown in Figure 1.

The simulation results demonstrate that newfal $(e, \alpha, \delta)$ exhibits better continuity and derivability than $\operatorname{fal}(e, \alpha, \delta)$. Thus, newfal $(e, \alpha, \delta)$ is not sensitive to the selection of the $\delta$ value and can avoid the high-frequency flutter phenomenon. In this study, the improved ADRC is designed based on newfal $(e, \alpha, \delta)$.

\subsection{Improved ADRC Design for the Current-Loop of the Pitch-} ing Axis of an Aerial Camera. Figure 2 shows the structure of the improved ADRC of the current-loop for the pitching axis control system of an aerial camera.
3.2.1. TD. $\mathrm{TD}$ in the improved $\mathrm{ADRC}$ is used to arrange the transient process. A continuous and differentiable input signal can be obtained by TD. Therefore, drastic changes in the control signal can be avoided effectively. The TD expression of the current-loop of the pitching axis control system of an aerial camera is designed as follows:

$$
\begin{aligned}
& \mathrm{fh}=\text { fhan }\left(z_{11}-i_{q}{ }^{*}, v_{2}, r_{0}, h_{0}\right), \\
& \dot{z_{11}}=v_{2}, \\
& \dot{v_{2}}=\mathrm{f} h,
\end{aligned}
$$

where $i_{q}{ }^{*}$ is the current given the quadrature axis, $z_{11}$ is the tracking signal for $i_{q}{ }^{*}, r_{0}$ is the velocity factor, $h_{0}$ is the filter 


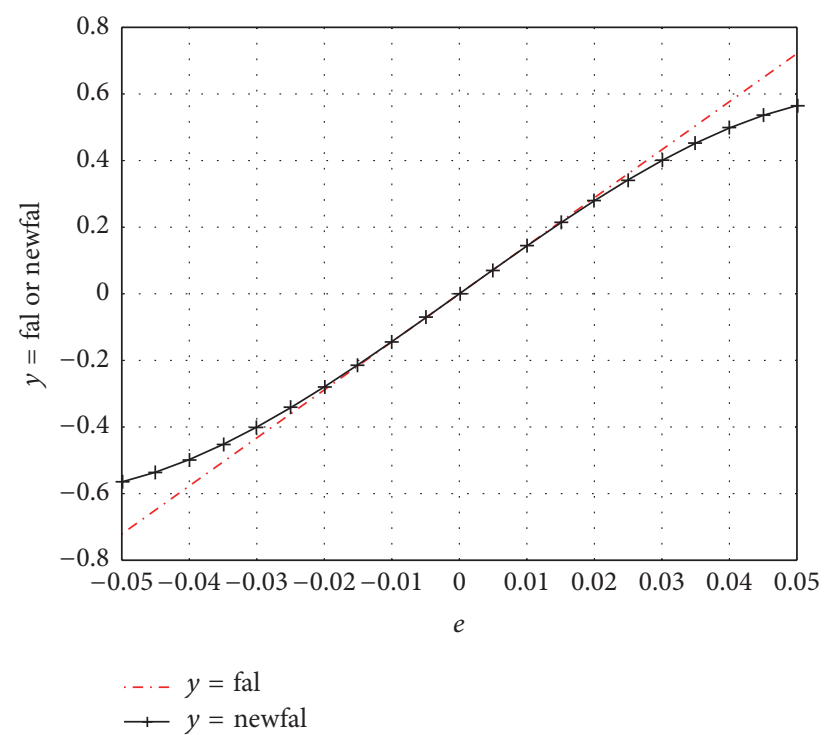

Figure 1: Curves of newfal $(\cdot)$ and fal $(\cdot)$ when $\alpha=0.05, \delta=0.06$.

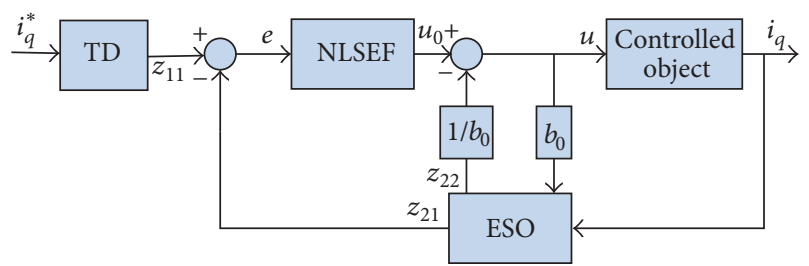

FIgURE 2: Structure of the improved ADRC of the current-loop of the pitching axis control system of an aerial camera.

factor, and $r_{0}$ and $h_{0}$ are used to arrange the speed of the transient process. fhan $\left(x_{1}, x_{2}, r_{0}, h_{0}\right)$ is an optimal control synthesis function and can be defined as

$$
\text { fhan }\left(v_{1}, v_{2}, r_{0}, h_{0}\right)=- \begin{cases}r_{0} \operatorname{sign}(\alpha), & |\alpha|>\delta \\ r_{0} \frac{\alpha}{\delta}, & |\alpha| \leq \delta,\end{cases}
$$

where

$$
\begin{aligned}
\alpha & = \begin{cases}v_{2}+\frac{\alpha_{0}-\delta}{{ }^{2}} \operatorname{sign}(y), & |y|>\delta_{0} \\
v_{2}+\frac{y}{h_{0}}, & |y| \leq \delta_{0},\end{cases} \\
\delta & =r_{0} h_{0}, \\
\delta_{0} & =\delta h_{0}, \\
y & =v_{1}+h_{0} v_{2}, \\
\alpha_{0} & =\sqrt{\delta^{2}+8 r_{0}|y|} .
\end{aligned}
$$

$v(t)$ is the input and $v_{1}$ is the output of the TD. $v_{1}$ can track $v(t)$ as fast as possible in the case of the acceleration constraint $|\ddot{v}| \leq r$. The larger the value of $r_{0}$, the faster the tracking speed. $v_{2}$ is the differentiation of $v(t)$ when $v_{1}$ is tracking $v(t)$. $h_{0}$ is

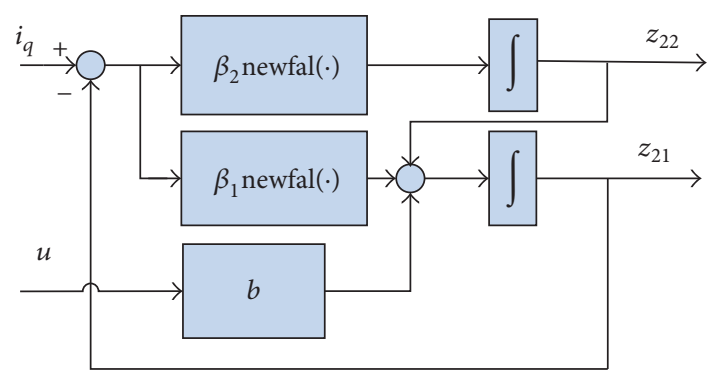

FIGURE 3: Internal structure of the improved ESO of the currentloop of the pitching axis of the aerial camera.

used to filter out the high-frequency interferences of $v(t)$. The value of $h_{0}$ is parameterized as $h_{0}=0.01$. The greater the value of $h_{0}$, the better the filtering effect.

3.2.2. Improved ESO. Improved ESO is the core of the improved ADRC, which is a nonlinear control technology that does not rely on the system model. Nonlinear dynamics, model uncertainty, and external disturbances are extended to a new state and observed by the improved ESO. The overtime variation of system can be predicted by the improved ESO. Therefore, the robustness of the system can be improved greatly. The expression of the improved ESO for the currentloop for the pitching axis control system of an aerial camera is designed as

$$
\begin{aligned}
& e_{1}=z_{21}-i_{q} \\
& \dot{z_{21}}=z_{22}-\beta_{1} \operatorname{newfal}\left(e_{1}, \alpha_{0}, \delta_{1}\right)+b_{0} u(t), \\
& \dot{z_{22}}=-\beta_{2} \operatorname{newfal}\left(e_{1}, \alpha_{1}, \delta_{1}\right),
\end{aligned}
$$

where $z_{21}$ is the tracking signal for $i_{q}, e_{1}$ is the tracking error, and $z_{22}$ is the tracking signal for the total disturbance $w(t)$. If the new nonlinear function newfal $(\cdot)$ is selected appropriately, the state variables $z_{21}$ and $z_{22}$ can indicate the state variables $i_{q}$ and $w(t)$, respectively. Therefore, the improved ESO for the current-loop for the pitching axis of the aerial camera can be obtained. $\beta_{1}, \beta_{2}$ are the gain coefficients of the improved ESO. $\alpha_{0}, \alpha_{1}$ are the nonlinear factors and satisfy the condition $0<\alpha_{1}<\alpha_{0} \leq 1 . \delta_{1}$ is a filtering factor with a value of $\delta_{1}=0.01 . b_{0}$ is the estimation of the compensation factor. The internal structure of the improved ESO of the current-loop for the pitching axis of the aerial camera is shown in Figure 3.

The stability of the improved ESO can be proved as follows. The bandwidth of the current-loop is defined as $\lambda$. The following equation can be obtained according to previous research:

$$
(s+\lambda)^{2}=s^{2}+\beta_{1} s+\beta_{2} .
$$

The nonlinear parts of the improved ESO are divided into many areas to analyze the stability of the improved ESO. All areas are replaced by piecewise linear functions. The stability 
of the entire area can be ensured when the stability of each area is ensured. The present study used the linear expression $z_{21}-i_{q}$ to replace the nonlinear function newfal( $\left.\cdot\right)$ to analyze the stability of the improved ESO. Therefore, (15) can be written as

$$
\begin{aligned}
& \dot{z_{21}}=z_{22}-\beta_{1}\left(z_{21}-i_{q}\right)+b_{0} u(t), \\
& \dot{z_{22}}=-\beta_{2}\left(z_{21}-i_{q}\right) .
\end{aligned}
$$

Laplace transform is carried out for the above equation and the following equation can be obtained:

$$
\begin{aligned}
& z_{21}(s) \cdot s=z_{22}(s)-2 \lambda z_{21}(s)+2 \lambda i_{q}(s)+b_{0} u(s), \\
& z_{22}(s) \cdot s=-\lambda^{2} z_{21}(s)+\lambda^{2} i_{q}(s) .
\end{aligned}
$$

therefore, the following equation can be obtained:

$$
\begin{aligned}
& z_{21}(s)=\frac{\lambda^{2}+2 \lambda s}{(s+\lambda)^{2}} \cdot i_{q}(s)+\frac{b_{0} s}{(s+\lambda)^{2}} \cdot u(s), \\
& z_{22}(s)=\frac{\lambda^{2} s}{(s+\lambda)^{2}} \cdot i_{q}(s)+\frac{\lambda^{2} b_{0}}{(s+\lambda)^{2}} \cdot u(s) .
\end{aligned}
$$

The errors of $z_{21}(s)$ and $z_{22}(s)$ can be calculated as follows:

$$
\begin{aligned}
& e_{1}(s)=z_{21}(s)-i_{q}(s), \\
& e_{2}(s)=z_{22}(s)-a(s)=z_{22}(s)-i_{q}(s)-b_{0} u(s) ;
\end{aligned}
$$

then, (21) can be calculated:

$$
\begin{aligned}
e_{1}(s)= & \frac{\lambda^{2}+2 \lambda s}{(s+\lambda)^{2}} \cdot i_{q}(s)+\frac{b_{0} s}{(s+\lambda)^{2}} \cdot u(s)-i_{q}(s), \\
e_{2}(s)= & \frac{\lambda^{2} s}{(s+\lambda)^{2}} \cdot i_{q}(s)+\frac{\lambda^{2} b_{0}}{(s+\lambda)^{2}} \cdot u(s)-i_{q}(s) \cdot s \\
& -b_{0} u(s), \\
e_{1}(s)= & -\frac{s^{2}}{(s+\lambda)^{2}} \cdot i_{q}(s)+\frac{b_{0} s}{(s+\lambda)^{2}} \cdot u(s), \\
e_{2}(s)= & -\frac{s^{2}+2 \lambda s}{(s+\lambda)^{2}} \cdot s \cdot i_{q}(s)-\frac{s^{2}+2 \lambda s}{(s+\lambda)^{2}} \cdot b_{0} \cdot u(s) .
\end{aligned}
$$

This article selected $i_{q}(t)$ and $u(t)$ as unit step input. Thus, $i_{q}(s)=1 / s$, and $u(s)^{q}=1 / s$. The steady-state errors can be calculated as follows:

$$
\begin{aligned}
& e_{s 1}=\lim _{s \rightarrow 0} s \cdot e_{1}(s)=0, \\
& e_{s 2}=\lim _{s \rightarrow 0} s \cdot e_{2}(s)=0 ;
\end{aligned}
$$

therefore, the improved ESO exhibits stability.

3.2.3. Improved NLSEF. Improved NLSEF is used to combine the state variables produced by tracking the TD and improved
TABLE 1: Parameters of the experimental permanent magnet synchronous motor.

\begin{tabular}{lcc}
\hline Parameter & Dimension & Numerical value \\
\hline Power supply voltage & VAC & 400 \\
Rated power & $\mathrm{W}$ & 2400 \\
Maximum nonload speed & $\mathrm{r} / \mathrm{min}$ & 2750 \\
Continuous current & $\mathrm{A}$ & 5.1 \\
Continuous torque & $\mathrm{N} \cdot \mathrm{m}$ & 6.11 \\
Peak current & $\mathrm{A}$ & 18 \\
Peak torque & $\mathrm{N} \cdot \mathrm{m}$ & 18 \\
Motor pole logarithmic & & 16 \\
Resistance & $\Omega$ & 2.9 \\
Inductance & $\mathrm{mH}$ & 6.8 \\
\hline
\end{tabular}

ESO with the error of estimated value nonlinearly. The expression of the improved NLSEF for the aerial camera pitching axis current-loop is designed as

$$
\begin{aligned}
& e_{2}=z_{11}-z_{21}, \\
& u_{0}=\beta_{3} \operatorname{newfal}\left(e_{2}, \alpha_{2}, \delta_{2}\right),
\end{aligned}
$$

where $\beta_{3}$ is the gain coefficient of the improved NLSEF, $\alpha_{2}$ is a nonlinear factor with a value between 0 and 1 , and $\delta_{2}$ is a filtering factor.

3.2.4. DCD. DCD is used to compensate for the total disturbance of the current-loop for the pitching axis of the aerial camera. The total disturbance is composed of internal disturbance, external disturbance, and coupling influence. The expression of the DCD for the current-loop of the pitching axis of the aerial camera is designed as

$$
u=u_{0}-\frac{z_{22}}{b_{0}}
$$

where $u_{0}$ is the control signal without DCD, $u$ is the control signal, and $z_{22} / b_{0}$ is the compensation component of total disturbance for the aerial camera pitching axis current-loop.

\section{Simulation Experiments and Discussion}

The improved ADRC is compared with the traditional ADRC and PI control strategy to accurately evaluate its performance. A PMSM is selected as the executing agency of the system. Table 1 shows the PMSM parameters, Table 2 presents the traditional ADRC parameters, and Table 3 presents the improved ADRC parameters.

Simulations are performed in the current-loop control system of the pitching axis of an aerial camera in the following three cases.

First, simulations are performed to verify the response speed and steady-state performance of the improved ADRC. A current of $1 \mathrm{~A}$ is applied on the rotor for nonload starting. Figure 4 shows the motor response curves under the PI control strategy, traditional ADRC, and improved ADRC. 
TABLE 2: Parameters of the traditional ADRC.

\begin{tabular}{lccc}
\hline $\begin{array}{l}\text { Component of } \\
\text { improved ADRC }\end{array}$ & Parameter name & Symbol & Numerical value \\
\hline TD & Velocity factor & $r_{0}$ & 10 \\
& Filter factor & $h_{0}$ & 0.02 \\
\hline & Nonlinear factor & $\alpha_{0}$ & 1 \\
& Nonlinear factor & $\alpha_{1}$ & 0.5 \\
Traditional ESO & Linear interval & $\delta_{1}$ & 0.01 \\
& width & $\beta_{1}$ & 60 \\
& Gain coefficient & $\beta_{2}$ & 1200 \\
\hline \multirow{3}{*}{ Traditional NLSEF } & Nonlinear factor & $\alpha_{2}$ & 0.5 \\
& Filter factor & $\delta_{2}$ & 0.01 \\
& Gain coefficient & $\beta_{3}$ & 75 \\
\hline \multirow{2}{*}{ DCD } & Compensation & $b_{0}$ & 1 \\
\hline
\end{tabular}

TABLE 3: Parameters of the improved ADRC.

\begin{tabular}{|c|c|c|c|}
\hline $\begin{array}{l}\text { Component of } \\
\text { improved ADRC }\end{array}$ & Parameter & Symbol & Numerical value \\
\hline \multirow{2}{*}{ TD } & Velocity factor & $r_{0}$ & 200 \\
\hline & Filter factor & $h_{0}$ & 0.01 \\
\hline \multirow{5}{*}{ Improved ESO } & Nonlinear factor & $\alpha_{0}$ & 0.5 \\
\hline & Nonlinear factor & $\alpha_{1}$ & 0.25 \\
\hline & $\begin{array}{c}\text { Linear interval } \\
\text { width }\end{array}$ & $\delta_{1}$ & 0.01 \\
\hline & Gain coefficient & $\beta_{1}$ & 30 \\
\hline & Gain coefficient & $\beta_{2}$ & 300 \\
\hline \multirow{3}{*}{ Improved NLSEF } & Nonlinear factor & $\alpha_{2}$ & 0.75 \\
\hline & Filter factor & $\delta_{2}$ & 0 \\
\hline & Gain coefficient & $\beta_{3}$ & 1000 \\
\hline DCD & $\begin{array}{c}\text { Compensation } \\
\text { coefficient }\end{array}$ & $b_{0}$ & 2.485 \\
\hline
\end{tabular}

The control strategy based on improved ADRC exhibits shorter response time and smaller overshoot amount than the PI control strategy and the traditional ADRC control strategy. The simulation results show that the overshoot amount of the control strategy based on improved ADRC is $14.2822 \%$ and 9.4183\% lower than those of the PI and traditional ADRC control strategies, respectively. The response time of the control strategy based on the improved ADRC is 1.5392 and 0.6459 s shorter than those of the PI and traditional ADRC control strategies, respectively. The current-loop parameters of the overshoot amount and the response times for the three strategies are shown in Table 4.

Second, simulations are performed to verify the antiinterference ability of the improved ADRC. As an external disturbance, a step signal of $1 \mathrm{~A}$ is applied on the motor for $3 \mathrm{~s}$. The current response curves are shown in Figure 5. The simulation results show that the improved ADRC control strategy exhibits smaller response curve fluctuation, shorter

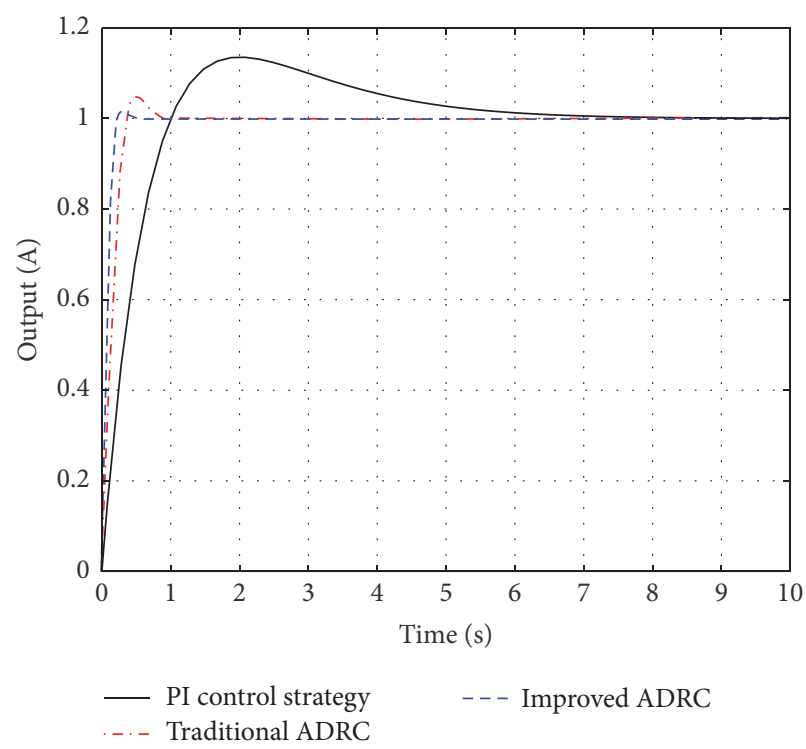

FigURE 4: Response curves of current-loop for nonload starting under the PI control strategy, traditional ADRC, and improved ADRC control strategy.

TABLE 4: Parameters of the overshoot amount and the response time for nonload starting under the PI control strategy, traditional ADRC, and improved ADRC control strategy.

\begin{tabular}{lcc}
\hline Control strategy & Response time/s & Overshoot amount \\
\hline PI & 7.0056 & $16.6352 \%$ \\
Traditional ADRC & 0.8623 & $5.7653 \%$ \\
Improved ADRC & 0.4564 & $2.3470 \%$ \\
\hline
\end{tabular}

TABLE 5: Parameters of the overshoot amount and recovery time under the PI control strategy, traditional ADRC, and improved ADRC control strategy for an external disturbance applying.

\begin{tabular}{lcc}
\hline Control strategy & Response time/s & Overshoot amount \\
\hline PI & 6.7281 & $40.2103 \%$ \\
Traditional ADRC & 0.4935 & $7.3252 \%$ \\
Improved ADRC & 0.4758 & $4.7341 \%$ \\
\hline
\end{tabular}

recovery time, and smaller load disturbance influence than the PI and traditional ADRC control strategies. The simulation results show that the overshoot amount of the improved ADRC control strategy is $7.5911 \%$ and $36.4762 \%$ lower than those of the traditional ADRC and PI control strategies. The recovery time of the improved ADRC control strategy is 1.4877 and $6.9423 \mathrm{~s}$ shorter than those of the traditional ADRC and PI control strategies. Table 5 presents the currentloop parameters of the overshoot amount and recovery time.

Finally, simulations are performed to verify the indicator ability of the improved ADRC. A sinusoidal signal input is applied to the current-loop control system. The current output curves for the traditional ADRC and improved ADRC control strategies are shown in Figure 6. According to the simulation results, the improved ADRC control strategy 


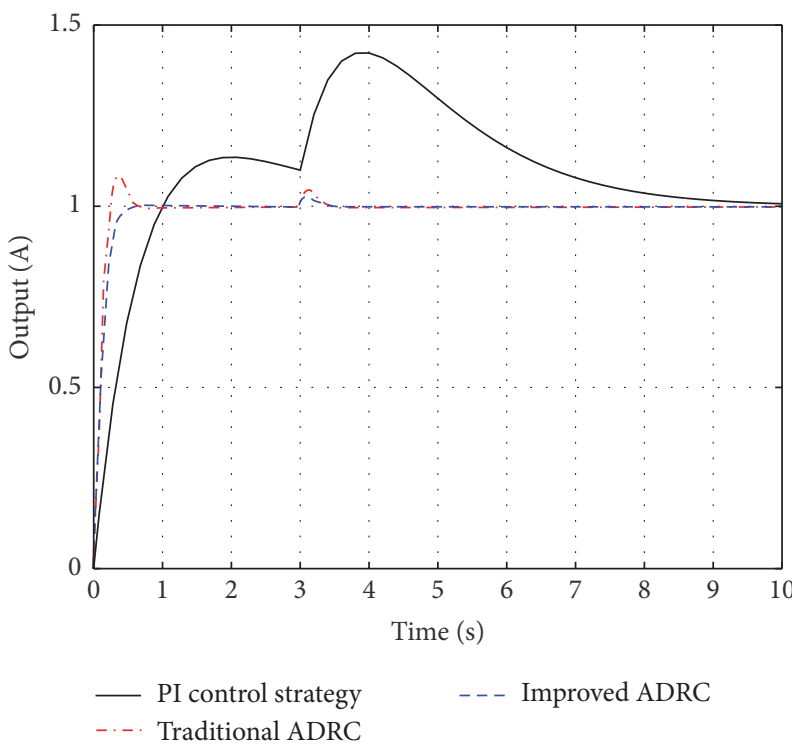

FIGURE 5: Response curves of current-loop for an external disturbance applying under the PI control strategy, traditional ADRC, and improved ADRC control strategy.

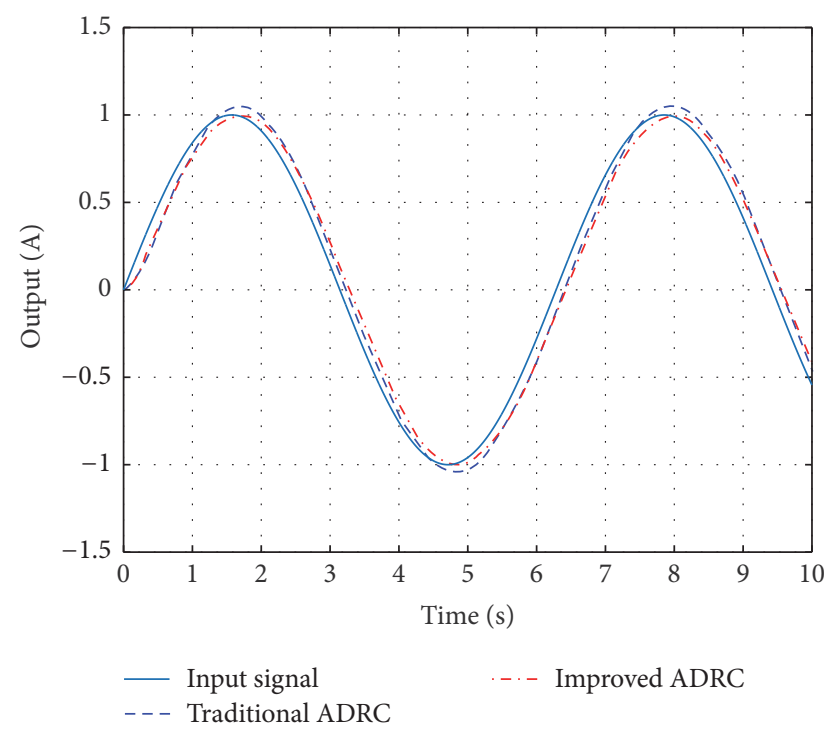

FIGURE 6: Response curves of the current-loop under the traditional ADRC and improved ADRC control strategies for a sinusoidal signal input.

exhibits better indicator ability than the traditional ADRC control strategy.

Simulations are also performed to verify the performance of $z_{22}$ tracking the disturbance, and the results are shown in Figure 7. Figure 7 shows that $z_{22}$ can track the disturbance in real time when a unit step disturbance is applied on the current-loop at the time $3 \mathrm{~s}$.

\section{Conclusions}

An improved ADRC of the current-loop of the pitching axis control system of an aerial camera is proposed. The

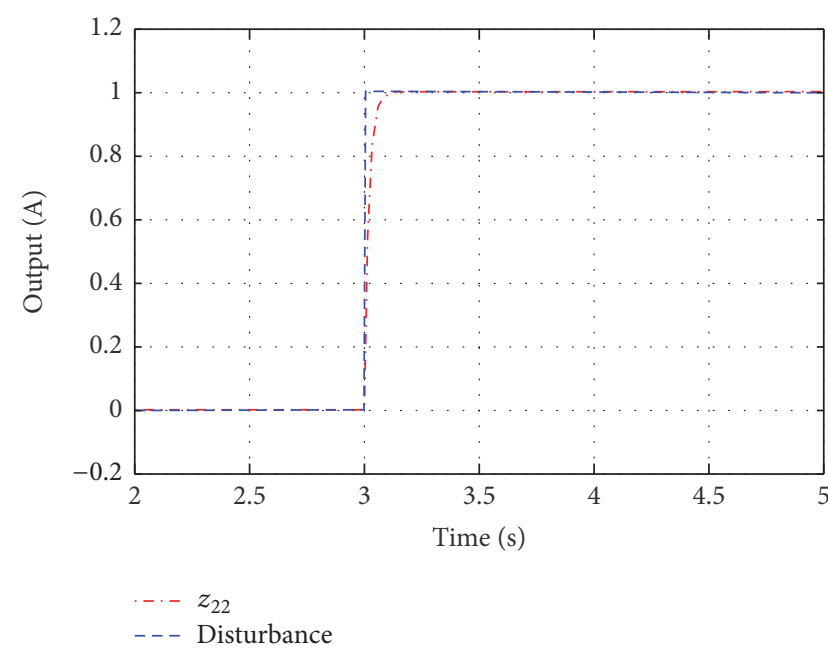

FIGURE 7: Response curves of $z_{22}$ and the disturbance.

new nonlinear function can effectively decrease the highfrequency flutter phenomenon, because it exhibits better continuity and smoothness than the previously available nonlinear functions. Therefore, the improved ADRC designed based on the new nonlinear function exhibits better control performances than the PI control strategy and the traditional ADRC. The nondifferentiable and discontinuous characteristics of the nonlinear function of NLSEF are overcome by the improved ADRC. Simulations are performed, and the results indicate that the system with improved ADRC control strategy exhibits better dynamic performance, static performance, and robustness than the system with PI control strategy and traditional ADRC control strategy. Extending the proposed design technique to the improved ADRC of the position-loop is an interesting topic, and this technique is the focus of our future study.

\section{Competing Interests}

The authors declare no conflict of interests regarding the publication of this paper.

\section{Acknowledgments}

This work was supported by the Key Project of Natural Science by Education Department of Anhui Province (no. KJ2015A316), the Outstanding Young Talents at Home Visit the School Training Project (no. gxfxZD2016101), and Natural Science Foundation of Anhui Province of China (nos. 1501021015 and 1408085ME105).

\section{References}

[1] M. Wang, Y.-F. Cheng, B. Yang, and X. Chen, "On-orbit calibration approach for star cameras based on the iteration method with variable weights," Applied Optics, vol. 54, no. 21, pp. 6425-6432, 2015.

[2] X. Guan and G.-T. Zheng, "Integrated design of space telescope vibration isolation and attitude control," Journal of Astronautics, vol. 34, no. 2, pp. 214-221, 2013. 
[3] A. A. A. Radwan and Y. A.-R. I. Mohamed, "Improved vector control strategy for current-source converters connected to very weak grids," IEEE Transactions on Power Systems, vol. 31, no. 4, pp. 3238-3248, 2016.

[4] B.-L. Liu, Y.-L. Wang, and Z.-J. Ding, "Active power filter optimal variable-structure current control strategy," Electrical Measurement and Instrumentation, vol. 51, no. 22, pp. 67-71, 2014.

[5] D. Novak and R. Riener, "Control strategies and artificial intelligence in rehabilitation robotics," Ai Magazine, vol. 36, no. 4, pp. 23-33, 2015.

[6] W. Liu, L. Luo, Z. Zhang, J. Xu, and Z. Huang, "An adaptive current control technique with constant frequency based on clocked signal," Automation of Electric Power Systems, vol. 38, no. 1, pp. 109-120, 2014.

[7] J. Han, "From PID to active disturbance rejection control," IEEE Transactions on Industrial Electronics, vol. 56, no. 3, pp. 900906, 2009.

[8] B.-Z. Guo and F.-F. Jin, "The active disturbance rejection and sliding mode control approach to the stabilization of the EulerBernoulli beam equation with boundary input disturbance," Automatica, vol. 49, no. 9, pp. 2911-2918, 2013.

[9] B.-Z. Guo and F.-F. Jin, "Sliding mode and active disturbance rejection control to stabilization of one-dimensional anti-stable wave equations subject to disturbance in boundary input," IEEE Transactions on Automatic Control, vol. 58, no. 5, pp. 1269-1274, 2013.

[10] B.-Z. Guo and H.-C. Zhou, "The active disturbance rejection control to stabilization for multi-dimensional wave equation with boundary control matched disturbance," IEEE Transactions on Automatic Control, vol. 60, no. 1, pp. 143-157, 2015.

[11] H. Sira-Ramírez, J. Linares-Flores, C. García-Rodríguez, and M. A. Contreras-Ordaz, "On the control of the permanent magnet synchronous motor: an active disturbance rejection control approach," IEEE Transactions on Control Systems Technology, vol. 22, no. 5, pp. 2056-2063, 2014.

[12] L. A. Castaneda, A. Luviano-Juarez, and I. Chairez, "Robust trajectory tracking of a delta robot through adaptive active disturbance rejection control," IEEE Transactions on Control Systems Technology, vol. 23, no. 4, pp. 1387-1398, 2015.

[13] M. Ran, Q. Wang, and C. Dong, "Stabilization of a class of nonlinear systems with actuator saturation via active disturbance rejection control," Automatica. A Journal of IFAC, the International Federation of Automatic Control, vol. 63, pp. 302310, 2016.

[14] W. Xue, W. Bai, S. Yang, K. Song, Y. Huang, and H. Xie, "ADRC with adaptive extended state observer and its application to airfuel ratio control in gasoline engines," IEEE Transactions on Industrial Electronics, vol. 62, no. 9, pp. 5847-5857, 2015.

[15] J. Li, Y.-Q. Xia, X.-H. Qi, Z.-Q. Gao, K. Chang, and F. Pu, "Absolute stability analysis of on-linear active disturbance rejection control for single-input-single-output systems via the circle criterion method," IET Control Theory \& Applications, vol. 9, no. 15, pp. 2320-2329, 2015.

[16] Z.-L. Zhao and B.-Z. Guo, "Active disturbance rejection control approach to stabilization of lower triangular systems with uncertainty," International Journal of Robust and Nonlinear Control, vol. 26, no. 11, pp. 2314-2337, 2016.

[17] B.-Z. Guo, Z.-H. Wu, and H.-C. Zhou, "Active disturbance rejection control approach to output-feedback stabilization of a class of uncertain nonlinear systems subject to stochastic disturbance," IEEE Transactions on Automatic Control, vol. 61, no. 6, pp. 1613-1618, 2016.

[18] F. Liu, Y. Li, Y. Cao, J. She, and M. Wu, "A two-layer active disturbance rejection controller design for load frequency control of interconnected power system," IEEE Transactions on Power Systems, vol. 31, no. 4, pp. 3320-3321, 2016.

[19] B. Li, Q. Hu, and G. Ma, "Extended State Observer based robust attitude control of spacecraft with input saturation," Aerospace Science and Technology, vol. 50, pp. 173-182, 2016.

[20] Z.-Q. Pu, R.-Y. Yuan, J.-Q. Yi, and X.-M. Tan, "A class of adaptive ESOs for nonlinear disturbed systems," IEEE Transactions on Industrial Electronics, vol. 62, no. 9, pp. 5858-5869, 2015.

[21] N.-M. Qi, C.-M. Qin, and Z.-G. Song, "Improved ADRC cascade decoupling controller design of hypersonic vehicle," Journal of Harbin Institute of Technology, vol. 43, no. 11, pp. 3438, 2011.

[22] W.-X. Xia, X.-D. Yang, and J.-H. Yan, "Application of improved ADRC technique in straight course error modeling of electromagnetic controlled gyrocompass," Electronics Optics \& Control, vol. 17, no. 6, pp. 73-79, 2010.

[23] L.-Y. Zhou and S.-J. Wang, "An improved ADRC based on nonlinear arctangent function," Shanghai Jiaotong Daxue Xuebao/Journal of Shanghai Jiaotong University, vol. 47, no. 7, pp. 1043-1048, 2013.

[24] M.-Y. Zhang, H.-B. Yang, J.-B. Zhang, T.-C. Ding, and H.-G. Jia, "Servo system of harmonic drive electromechanical actuator using improved ADRC," Optics and Precision Engineering, vol. 22, no. 1, pp. 99-108, 2014.

[25] Y. Shen, K. Shao, W. Ren, and Y. Liu, "Diving control of autonomous underwater vehicle based on improved active disturbance rejection control approach," Neurocomputing, vol. 173, pp. 1377-1385, 2016.

[26] X. Yang, J. Cui, D. Lao, D. Li, and J. Chen, "Input Shaping enhanced Active Disturbance Rejection Control for a twin rotor multi-input multi-output system (TRMS)," ISA Transactions, vol. 62, pp. 287-298, 2016. 


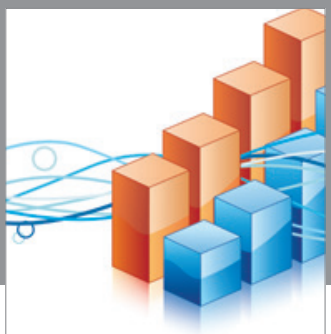

Advances in

Operations Research

vatem alat4

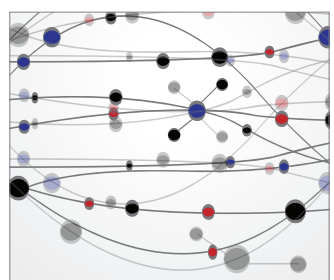

\section{The Scientific} World Journal
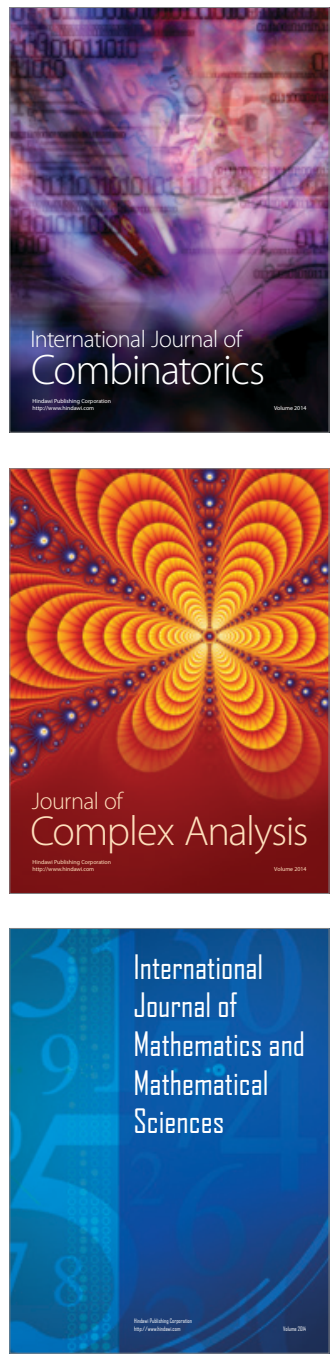
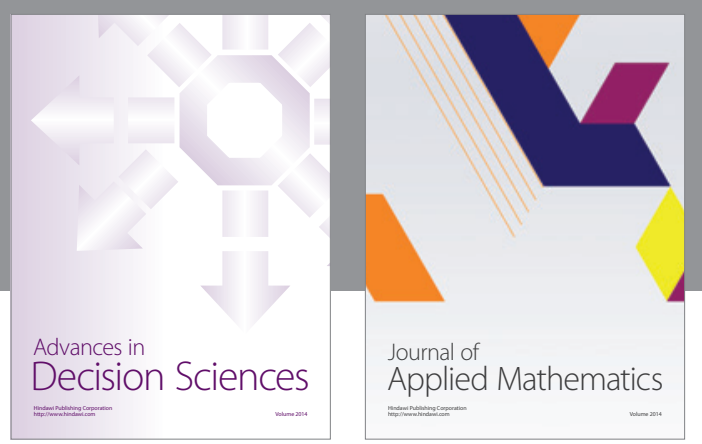

Algebra

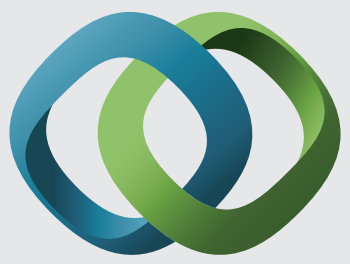

\section{Hindawi}

Submit your manuscripts at

https://www.hindawi.com
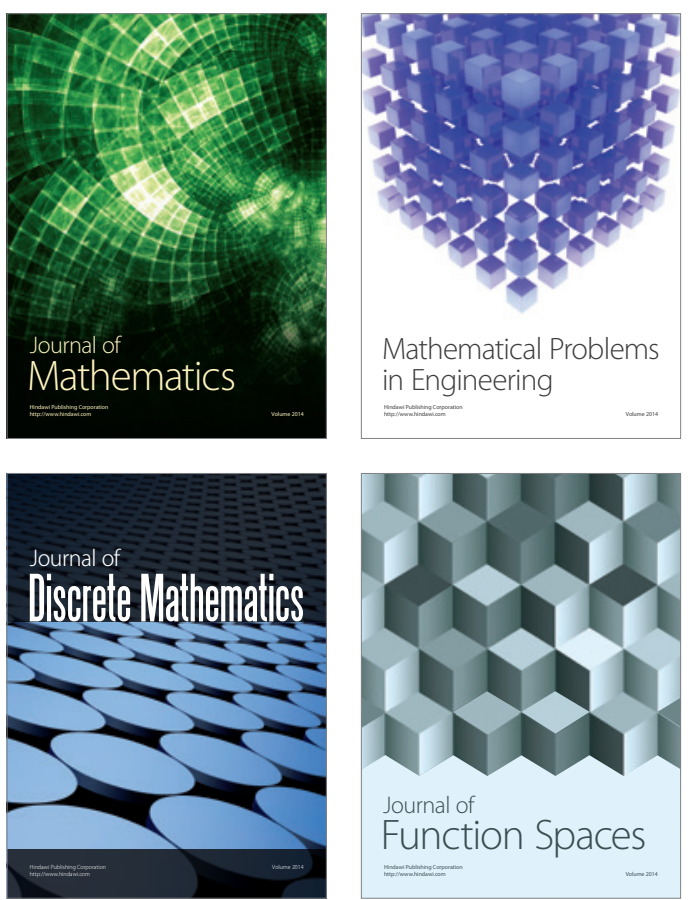

Mathematical Problems in Engineering
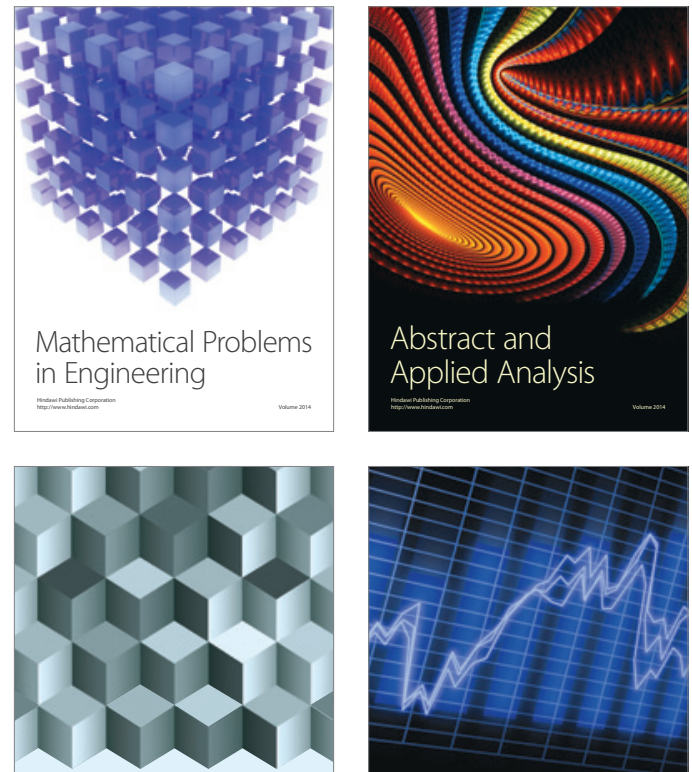

Journal of

Function Spaces

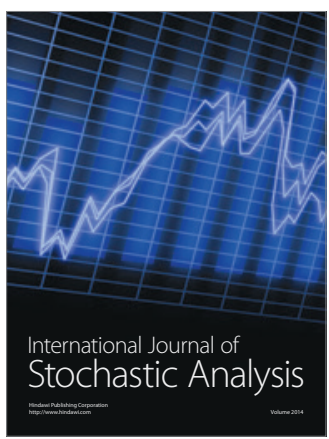

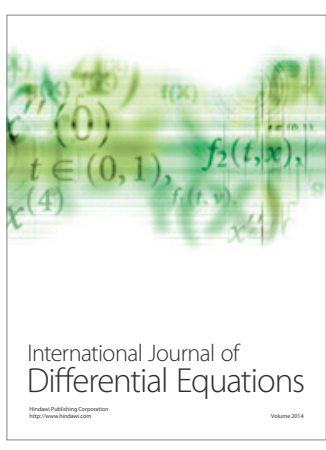
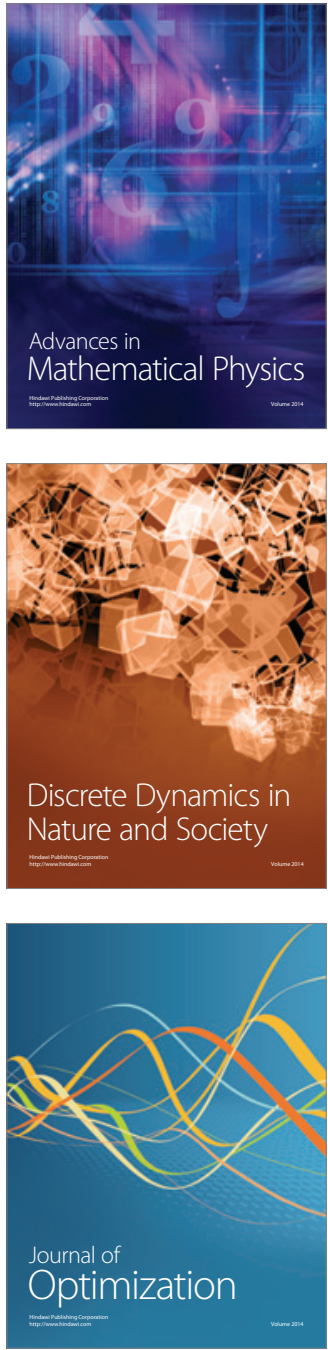